\title{
Thermodynamics of ordered and disordered phases in the binary Mo-Ru system
}

\author{
Kissavos, A.E.; Shallcross, Sam; Kaufman, L.; Granas, O.; Ruban, Andrei; Abrikosov, I.A.
}

Published in:

Physical Review B Condensed Matter

Link to article, DOI:

10.1103/PhysRevB.75.184203

Publication date:

2007

Document Version

Publisher's PDF, also known as Version of record

Link back to DTU Orbit

Citation (APA):

Kissavos, A. E., Shallcross, S., Kaufman, L., Granas, O., Ruban, A., \& Abrikosov, I. A. (2007). Thermodynamics of ordered and disordered phases in the binary Mo-Ru system. Physical Review B Condensed Matter, 75(18), 184203. https://doi.org/10.1103/PhysRevB.75.184203

\section{General rights}

Copyright and moral rights for the publications made accessible in the public portal are retained by the authors and/or other copyright owners and it is a condition of accessing publications that users recognise and abide by the legal requirements associated with these rights.

- Users may download and print one copy of any publication from the public portal for the purpose of private study or research.

- You may not further distribute the material or use it for any profit-making activity or commercial gain

- You may freely distribute the URL identifying the publication in the public portal 


\title{
Thermodynamics of ordered and disordered phases in the binary Mo-Ru system
}

\author{
A. E. Kissavos* \\ Department of Physics, Chemistry and Biology (IFM), University of Linköping, SE-581 83 Linköping, Sweden \\ S. Shallcross \\ Department of Physics, Technical University of Denmark, Building 309-307-312, DK-2800 Kongens Lyngby, Denmark
}

L. Kaufman

CALPHAD, Inc., 140 Clark Road, Brookline, Massachusetts 02445-5848, USA

O. Grånäs

Department of Physics, Condensed Matter Theory Group, P.O. Box 530, SE-751 21 Uppsala, Sweden

A. V. Ruban

Applied Material Science, Royal Institute of Technology, Brinellvägen 23, 10044 Stockholm, Sweden

I. A. Abrikosov

Department of Physics, Chemistry and Biology (IFM), University of Linköping, SE-581 83 Linköping, Sweden (Received 7 November 2006; revised manuscript received 16 February 2007; published 15 May 2007)

\begin{abstract}
We have performed $a b$ initio calculations of the mixing enthalpy for the Mo-Ru alloy system. Both completely random alloys on the fcc, bcc, and hcp lattices as well as ordered and partially ordered structures based on the hcp lattice and a $\sigma$ phase have been examined. Further, we have performed a ground-state search for the $\mathrm{Ru}$-rich region using $a b$ initio derived effective interactions, and find a series of structures below the tie line of the simple compounds. Using the structures from this ground-state search, we are able to make an estimation of the contribution to the total energy due to ordering effects in this system. We find unusually large deviations between calculated and experimental values of the mixing enthalpy for Ru-rich hcp alloys. Our calculations indicate, in agreement with experiment, that there are ordering trends in the system. However, even under assumption of maximal order theoretical results differ substantially from the experiment. Possible reasons for the disagreement are discussed.
\end{abstract}

DOI: $10.1103 /$ PhysRevB.75.184203

PACS number(s): 61.66.Dk, 71.23.-k, 71.20.Be, 71.15.Nc

\section{INTRODUCTION}

Due to the increasing efficiency and accuracy of ab initio methods for the electronic structure and total-energy calculations based on the density-functional theory (DFT), ${ }^{1,2}$ theoretically obtained results have become a reliable source of information with often equally good quality as experimentally measured results. We can presently see an emergence of theoretically derived databases, ${ }^{3-5}$ where both experimental results can be verified or challenged, but also quantities which are experimentally hard to measure are being published. There are also discussions on the possibility to merge $a b$ initio techniques with the empirical CALPHAD (computer coupling of phase diagrams and thermochemistry) methodology (Ref. 6) in order to improve on the predictive capacity of the latter. ${ }^{7-9}$

The future use of these theoretical databases in practical applications depends crucially on the consistency between theoretical and experimental results, since one must be able to use the different sets of data complimentary. In many cases nowadays, there is rather good agreement between calculated and experimental thermodynamic properties of metals and alloys, such as lattice parameters, bulk moduli, and mixing energies. In some cases though, the discrepancy is significant, and they attract particular attention because of a suspicion of the major failures of the underlying approxima- tions used by the theory. For example, large difference between the calculated and experimental volumes of $\mathrm{Pu}$ (in the nonmagnetic phase) has inspired an intensive research. ${ }^{10} \mathrm{An}$ other critical test of the theory is the prediction of structural energy differences for some transitional elements. ${ }^{11}$ In particular, Mo has been investigated in detail. ${ }^{12,13}$ The ab initio predictions have in general given a much higher value for the energy difference between the closed-packed [face-centered cubic (fcc) or hexagonal closed packed (hcp)] and more open body-centered cubic (bcc) structures of Mo than the values extrapolated from the experimental data by means of the CALPHAD methods. Indeed, the accepted CALPHAD value of the energy difference between the hcp and bcc structures is 9 mRy. ${ }^{14}$ This is considerably less than the ab initio values which range between 35 and $50 \mathrm{mRy}$, depending on the approximations used for the simulations. ${ }^{7,11,15-19}$ Here, Grimvall et al. ${ }^{12}$ and Persson et al. ${ }^{20}$ have suggested that dynamical instability of the hcp Mo plays a decisive role.

In this paper, we investigate in detail another case of the remarkable disagreement between theoretically obtained values for the thermodynamic properties and their experimental values, which was observed recently. It is strongly believed nowadays that theoretically predicted energies of formation of the intermetallic compounds and mixing energies of alloys can be calculated with very high accuracy. ${ }^{3-5,9}$ On the contrary, Kissavos et al. ${ }^{7}$ and later Shin et al. ${ }^{8}$ obtained very 
large, about $10 \mathrm{mRy}$ per atom or factor of 5 , difference between the calculated and experimental values of the mixing enthalpy for the Ru-rich Mo-Ru alloys.

In an earlier paper, ${ }^{7}$ it was suggested that the error might be traced back to the problem of the structural energy difference in Mo. The trick used in that paper was to separate the Mo structural energy difference $E_{\text {struc }}^{\mathrm{Mo}}$ from the mixing enthalpy by noting that the mixing enthalpy of the Mo-Ru solid solution can be expressed as a sum of two terms: the mixing enthalpy on a fixed hcp lattice $H_{h c p}$ and a term arising from the energy cost of changing bcc Mo into the hcp structure of the solution lattice. This latter term is just a product of the alloy concentration and $E_{\text {struc }}^{\mathrm{Mo}}$.

$$
H_{\mathrm{Mo}_{c} \mathrm{Ru}_{1-c}}=E_{\mathrm{Mo}_{c} \mathrm{Ru}_{1-c}}^{\text {alloy }}-c E_{b c c}^{\mathrm{Mo}}-(1-c) E_{h c p}^{\mathrm{Ru}}=H_{h c p}+c E_{\text {struc }}^{\mathrm{Mo}} .
$$

Assuming that the mixing enthalpy on a fixed lattice can be treated reliably with the ab initio methods, which is often the case, ${ }^{21,22}$ any substantial difference between $a b$ initio and experimental values of the mixing enthalpy may be expected to mainly originate from a possible failure in the $a b$ initio description of the Mo structural energy difference. Further, the simple relation given by Eq. (1) allows one to treat $E_{\text {struc }}^{\mathrm{Mo}}$ as a free parameter and one could use instead the CALPHAD value here. In this way, and under the assumption that $H_{h c p}$ is calculated accurately, Kissavos et al. ${ }^{7}$ were able to reproduce the experimental mixing enthalpy of Ru-rich hcp alloys.

While the results seems to indicate that the use of $a b$ initio mixing enthalpies calculated at fixed underlying crystal lattice together with CALPHAD structural energy differences restore the agreement between theory and experiment, the investigation of the alloy thermodynamics for the binary Mo-Ru system presented in Ref. 7 was not complete. Especially, the effects due to possible ordering were not thoroughly investigated. In this paper, we examine completely random alloys on the fcc, bcc, and hcp lattices as well as ordered and partially ordered structures based on the hcp lattice and a complex ordered $\sigma$ phase. The paper is organized as follows. In Sec. II we describe in more detail the computational methods that will be used in this work. In Sec. III we present our results for the mixing enthalpies of completely random alloys. In Sec. IV we investigate ordering effects in Mo-Ru system and describe the details of a ground-state search performed for Ru-rich alloys. Mixing enthalpies of ordered structures are presented in Sec. V. Thereafter, we end the paper with a discussion in Sec. VI and conclusions in Sec. VII.

\section{COMPUTATIONAL METHOD}

The $a b$ initio calculations were performed using DFT (Refs. 1 and 2) and the exact muffin-tin orbital (EMTO) method. ${ }^{23,24}$ For the calculation of total energies, we used the full charge density (FCD) technique, whereby a muffin-tin approximation is used for the density in the self-consistent cycle followed by an evaluation of the total energy using the full (nonspherical) charge density. ${ }^{25}$ When combined with the FCD method, the EMTO method has an accuracy similar to that achieved with full-potential methods. ${ }^{26,27}$ For the calculation of random alloys we used the coherent potential approximation $^{28}$ (CPA) with an electrostatic correction to the one electron potential. ${ }^{29-31}$ Within the EMTO method, the CPA was implemented in Ref. 32 and it was shown in an earlier work that the FCD-EMTO-CPA method gives a very good description of substitutional random alloys. ${ }^{32,33}$

The density-functional calculations were performed within the generalized gradient approximation of PerdewBurke-Ernzerhof ${ }^{34}$ for the exchange-correlation contribution to the total energy and one-electron potential. All total energies were calculated at the theoretical equilibrium volumes for the corresponding phase. Deviations from the ideal $c / a$ ratio in the hcp structure were investigated and found to give very small contributions to the energy (the maximum deviation being $0.2 \mathrm{mRy}$ ), so ideal $c / a$ ratio was used throughout. The EMTO basis set included $s, p, d$, and $f$ orbitals and the angular momentum cutoff for the FCD calculations was $l_{\max }=11$. The valence electrons were treated scalar relativistically, with the core charge density recalculated at each iteration. For the k space integration, 506, 916, 891, 2640, and $2200 \mathbf{k}$ points distributed according to the Monkhorst-Pack technique were used in the irreducible zones of the bcc, fcc, hcp, $D O_{19}$, and $B 19$ structures, respectively.

For the simulations of the $\sigma$ phase, and to estimate the contribution to the total energy of random alloys due to local lattice relaxations, we used a full-potential projector augmented wave (PAW) method ${ }^{35}$ implemented in the Vienna $a b$ initio simulation package (VASP), ${ }^{36-38}$ generally known to be among the most accurate methods for electronic structure calculations. The plane-wave energy cutoff was set to $300 \mathrm{eV}$ for both the random alloy and the $\sigma$-phase calculations. The relaxation of the disordered hcp phase was performed using a supercell constructed as special quasirandom structure (SQS). ${ }^{39}$ The SQS with 54 atoms simulating $\mathrm{Mo}_{0.26} \mathrm{Ru}_{0.74}$ alloy was constructed, as described in Ref. 40. The number of bands used was 384; the Brillouin-zone integration was done using $98 k$ points in the irreducible zone distributed according to the Monkhorst-Pack scheme. The $\sigma$-phase calculations were performed with 228 to 192 bands and $112 k$ points in the irreducible part of the Brillouin zone.

\section{MIXING ENTHALPIES OF RANDOM ALLOYS}

The mixing enthalpies for Mo-Ru calculated for the completely random bcc, fcc, and hcp alloys are presented in Fig. 1. The results of Shin et $a .^{8}$ are also included in the figure. The two calculations produce results which are in close agreement to each other, if one compares the curves calculated for ideal (undistorted) underlying crystal lattices. The difference increases with increasing Mo concentration, and the largest difference is obtained for pure Mo, which is more than $5 \mathrm{mRy}$. We therefore conclude that the main source for the difference can be traced back to a well-known spread of the first-principles results for the structural energy difference in pure Mo, ${ }^{7,11}$ rather than to the different ways of the simulation of the disorder effects used by us (CPA) and in Ref. 8 (supercell approach). Most importantly is that for the Ru-rich alloys, the two theoretical curves are very close to each other. 


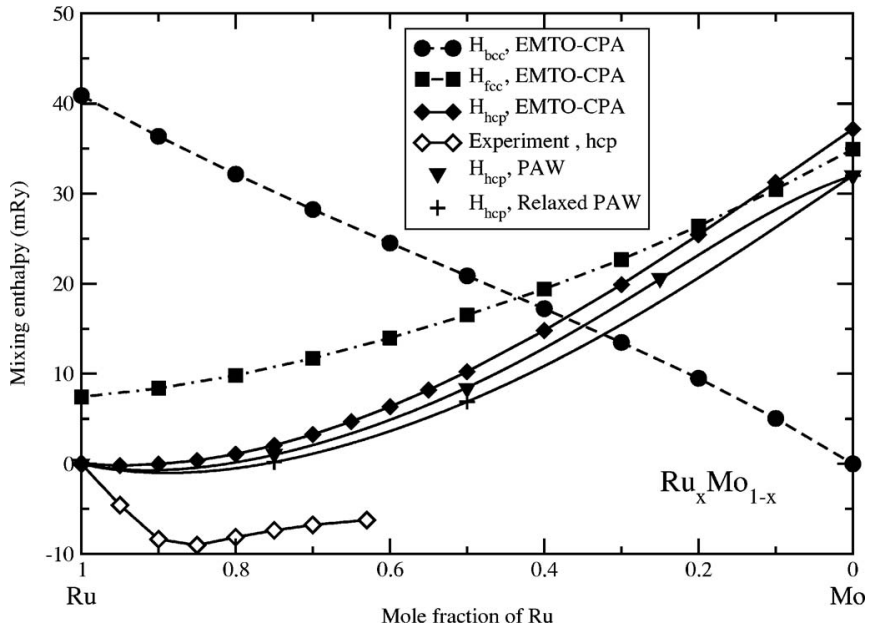

FIG. 1. Mixing enthalpy for random $\mathrm{Ru}_{x} \mathrm{Mo}_{1-x}$ alloys. Results calculated in this work by means of EMTO-CPA method for bcc (filled circles), fcc (filled squares), and hcp (filled diamonds) alloys are shown together with experimental data for Ru-rich hcp alloys from Ref. 41 (open diamonds). Theoretical results for hcp Mo-Ru alloys simulated by supercells with atoms distributed over sites of ideal underlying crystal lattice (filled triangles) and with fully relaxed atomic positions (crosses) obtained by PAW method in Ref. 8 are also shown for comparison.

On the contrary, as can be seen in Fig. 1, the calculated mixing enthalpy for the Ru-rich hcp alloys strongly disagrees with the available experimental data. ${ }^{41}$ The difference can be due to inaccuracy of the theoretical simulations, or because of the difficulty to measuring the mixing energy for refractory materials. Let us first analyze the limitations of the presented theoretical results. There are several effects that could be responsible for the disagreement. First, in a real alloy, there may be important lattice relaxation effects, and these are completely missed by the CPA due to the approximation of the fixed underlying crystal lattice. Second, the effects of the chemical short-range order are also completely neglected by the CPA because of its single site nature. Also, the longrange order might contribute significantly to the mixing enthalpy. Last, it could be that the structural energy term is responsible for the mismatch between experimental and theoretical results, as explained in the Introduction.

We first tackle the question of the relaxation energy. For all the systems in the present study, the total energy was minimized with respect to volume. We also checked that the deviations of $c / a$ ratio from its ideal value in the hcp phase have minimal effect on the total energies (the maximum deviation being $0.2 \mathrm{mRy}$ ). Thus, we conclude that in Mo-Ru random alloys, the only important relaxation term comes from local lattice relaxations. These occur because at any given site, the point group symmetry will in general be lower than the symmetry of the underlying lattice. For example, a Mo site with both Ru and Mo neighbors can lack the inversion symmetry that the parent lattice has.

Recently, a simple model has been proposed that allows one to quantitatively determine the contribution to the alloy total energy of this effect without doing expansive supercell calculations, the effective tetrahedron model. ${ }^{42}$ In the effec- tive tetrahedron model (ETM), the assumption is that the relaxation energy of the smallest cluster at the underlying alloy lattice is a function of the change in volume of that cluster. The relaxation energies obtained within the ETM for the interesting concentration range are, as expected, small. The maximum value given by the ETM is $0.3 \mathrm{mRy} / \mathrm{atom}$ around a concentration of $75 \% \mathrm{Ru}$. Our test calculation for this quantity performed using a 54 atom SQS, and the PAW method as implemented in VASP gave a result of $0.86 \mathrm{mRy}$ at a concentration of $74 \% \mathrm{Ru}$. A few more points were calculated by Shin et al. ${ }^{8}$ and can be seen in Fig. 1.

One can also notice that certain carefulness must be exhibited when using supercell techniques for finding relaxation energies. If the atoms relax too far and in a collective manner, that is, if they actually try to find positions which correspond to another structure rather than the structure that one starts from, the calculated relaxation energy might be irrelevant for the analysis of the energetics of the original structure. This, for example, may happen in an attempt to perform a structural optimization on the dynamically unstable systems, such as the hcp Mo-Ru alloys at high Mo concentrations. Even though one imposes a shape constraint on the supercell in order to keep the structure, a use of large supercells may lead to substantial reconstructions of the structure inside the "bulk" of the supercell where a new structure develops. As a matter of fact, the problem was also realized by Shin et al. who found that this actually happened for an alloy with Mo concentration of $75 \%$ (even though they used relatively small supercells). Since we are interested in the mixing enthalpy for the particular structure, we really want an estimate of the relaxation energy for the lattice in question, rather than the total energy for another lattice.

It is also obvious that the ETM cannot be used for the prediction of the relaxation energies in cases where the lattice is much distorted, which would be the case in a dynamically unstable system. However, our relaxed hcp supercell at $74 \% \mathrm{Ru}$ is still within the stability field for the hcp structure, and we can trust our supercell results. At the same time, the unusually big difference between the ETM and the supercell estimates of the relaxation energy may be due to the fact that the system approaches the dynamical instability.

It is important to point out that although the discrepancy between the ETM and the SQS results are unusually large for so similarly sized atoms as $\mathrm{Ru}$ and Mo, nevertheless the energy contribution due to the local lattice relaxations is small compared to the relative energy range in question (e.g., the difference between the theoretical and experimental results). We therefore do not consider lattice relaxation effects for the rest of this paper, unless the opposite is explicitly specified.

\section{ORDERING IN Mo-Ru SYSTEM}

\section{A. hcp alloys: Effective interactions}

In order to estimate the maximal possible error of the calculated mixing enthalpies for the hcp Ru-rich Mo-Ru alloys due to the neglect of the short-range order effects in the CPA, we decided to find the lowest-energy ordered states in this region of the concentrations. To be able to perform this 


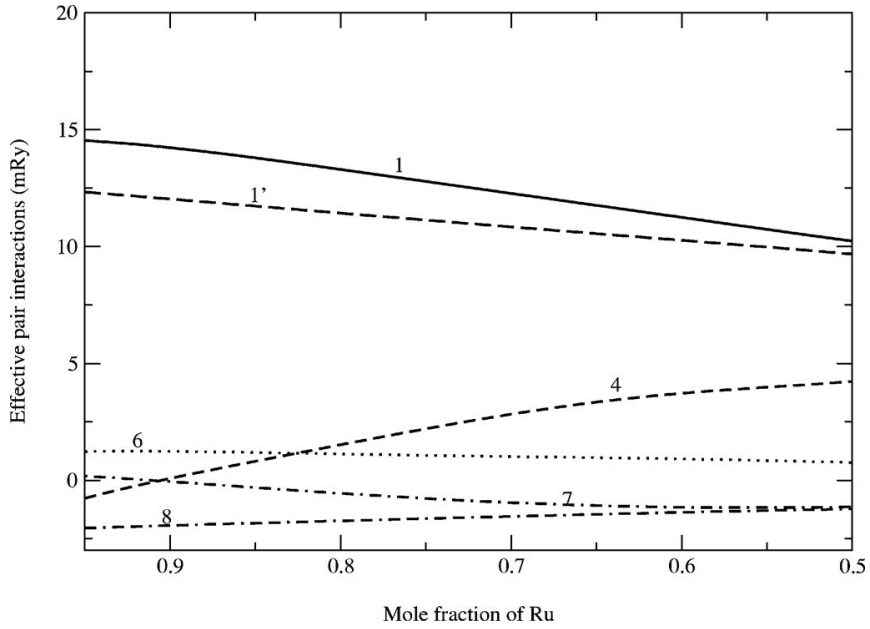

FIG. 2. Effective pair interactions of the Ising Hamiltonian determined for the hcp Ru-Mo alloys by the screened generalized perturbation method. The number stands for the shell to which the interactions belong. For the hcp lattice, we have two inequivalent first nearest-neighbor shells, denoted 1 and $1^{\prime}$ in the figure, respectively.

so-called ground-state search, which we present in the next section, one needs to determine the effective cluster interactions of a classical Ising Hamiltonian. ${ }^{43}$ The idea with getting these interactions is to minimize the energy of relatively large supercells with respect to the site occupation using the Ising Hamiltonian rather than by the direct electronic structure calculations. We can then use these possibly new ground states as an estimate of the maximal possible energy contribution due to the ordering effects in the Mo-Ru system. Of course, the structures presented in the next section may not be the real ground-state structure, since we impose restraints on the search by our choice of relatively small supercells, and also because of the neglect of the lattice relaxation effects. However, in any case their mixing enthalpies will give us a very good indication of the maximal possible energy gain the ordering can give because there are no ordered structures observed in Ru-rich Mo-Ru alloys experimentally. Also, an attempt to predict an exact ground state in such a system as Mo-Ru is to a very large degree a meaningless task because the diffusion is blocked at very high temperature.

In this work, we choose to determine the effective cluster interactions by means of the screened generalized perturbation method, ${ }^{44,45}$ which has been proven to have quantitative accuracy for the chemical part of the alloy interactions. Since the relaxation effects in this system are small on the energy scale relevant for our study, our effective Hamiltonian should be quite accurate. The screened generalized perturbation method (SGPM) interactions consist of two terms. First, the "bare" interactions calculated with the usual GPM formula, ${ }^{44,45}$ and then one has to add to the bare interactions a contribution which results from the screening of the embedded cluster by the effective medium, which represent the alloy on the average. ${ }^{31}$

The SGPM pair interactions calculated in this way are shown in Fig. 2. Higher-order terms, three-body, four-body, and biquadratic interactions were also calculated and found to be negligible. Clearly, there is very little concentration or volume dependence of the nearest-neighbor interactions in this system. However, the albeit not very pronounced concentration dependence of the fourth nearest-neighbor interaction turns out to have a quite significant effect on the groundstate structures as will be discussed in the next section.

In order to test the quality of our interactions, we have calculated $a b$ initio ordering energies of several ordered and partially ordered alloys. We have chosen the $D O_{19}, B 19$, $\mathrm{CuPt} \mathrm{I}$, and $A B$ structures. $D O_{19}$ and $B 19$ correspond to the $L 1_{2}$ and $L 1_{0}$ structures respectively, of the the better known fcc system. ${ }^{43}$ We have chosen this set of structures as they span a wide range of energies. A comparison between the $a b$ initio and SGPM calculated values is presented in Table I. It can be seen that the agreement between both approaches is generally excellent, in particular, for the $D O_{19}$ structure where the error is always less than $0.1 \mathrm{mRy}$. However, for the $B 19$ and $\mathrm{CuPt}$ I structures, the error is larger taking its maximum value at the stoichiometry of the structure of $1.0 \mathrm{mRy}$. The source of this error is most likely specific electrostatic effects associated with the lower symmetry of the $B 19$ and CuPt I structures.

\section{B. Ground-state search}

Recently, there was a great deal of interest generated by the observation of a series of so-called adaptive structure found as the ground states of fcc (Ref. 46) and bcc (Ref. 47) binary alloys. In Ref. 46, these were attributed to a longrange strain interaction, while in Ref. 47, these structures were observed arising from only chemical interactions. Using the effective interactions discussed in the previous section, we now present a ground-state search for the Ru rich

TABLE I. Ordering energies (in mRy/atom) calculated directly from the EMTO-CPA method (left-hand column at each concentration) and via the effective interactions from the screened generalized perturbation method (right-hand column).

\begin{tabular}{|c|c|c|c|c|c|c|c|c|}
\hline \multirow[b]{2}{*}{ Structure } & \multicolumn{2}{|c|}{$0.95 \% \mathrm{Ru}$} & \multicolumn{2}{|c|}{$0.75 \% \mathrm{Ru}$} & \multicolumn{2}{|c|}{$0.40 \% \mathrm{Ru}$} & \multicolumn{2}{|c|}{$0.50 \% \mathrm{Ru}$} \\
\hline & Direct & SGPM & Direct & SGPM & Direct & SGPM & Direct & SGPM \\
\hline$D O_{19}$ & -2.064 & -2.236 & -4.638 & -4.758 & -2.178 & -2.259 & -1.290 & -1.333 \\
\hline$B 19$ & -0.635 & -0.745 & -1.376 & -1.586 & -2.508 & -3.012 & -3.003 & -4.001 \\
\hline $\mathrm{CuPt} \mathrm{I}$ & -0.277 & -0.352 & -0.791 & -1.040 & -2.123 & -2.732 & -3.222 & -3.882 \\
\hline$A B$ & -0.07 & -0.114 & -0.098 & -0.177 & -0.075 & -0.222 & -0.103 & -0.169 \\
\hline
\end{tabular}




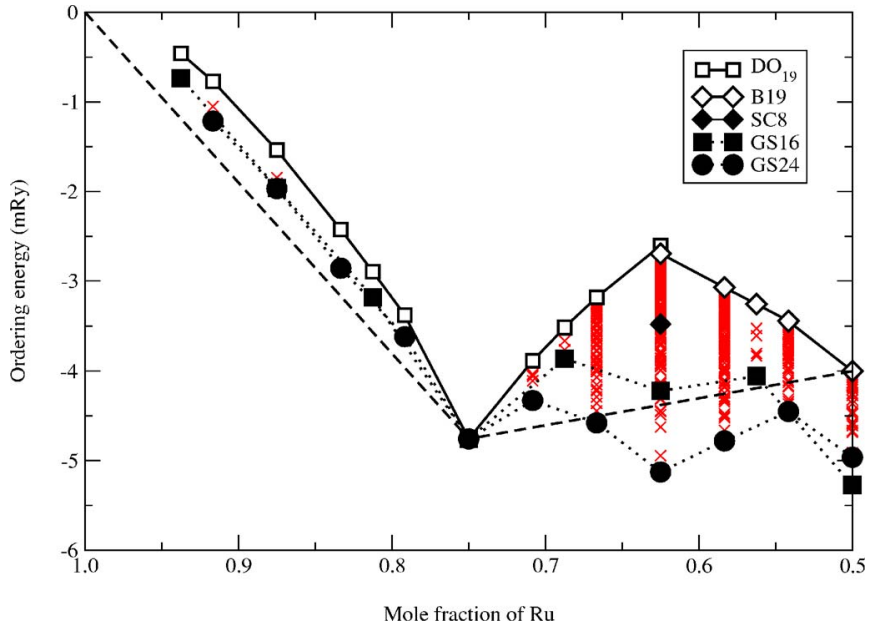

FIG. 3. (Color online) Results of a ground-state search for in Ru-rich hcp region. Shown are results for ordered and partially ordered $D O_{19}$ (open squares) and $B 19$ (open diamonds) alloys, as well as for the lowest energy structures based on 8 (filled diamonds), 16 (filled squares), and 24 (filled circles) atom supercells at each concentration. Crosses represent the energies of many different configurations possible in the large supercells (see text for more explanation).

concentrations of the Mo-Ru system. Since there is in any case no ordering transition experimentally observable in this system, the purpose of such a ground-state search is simply to complement the results for fcc and bcc lattices of previous works, and to highlight what may happen in other hcp-based binary alloy systems.

In Fig. 3 we present the results of an exhaustive groundstate search for supercells consisting of 8,16 , and 24 atoms. The supercell structure that we use is simply that of the $D O_{19}$ structure extended in the $z$ axis direction to include more (111) planes. Interestingly, we find that while at $75 \% \mathrm{Ru}$ the $D O_{19}$ structure has the lowest energy, closer to $50 \% \mathrm{Ru}$, we find a series of long period structures having an energy below the tie line connecting the $D O_{19}$ and $B 19$ structures.

We have tested that supercells based on other geometries do not provide any new ground-state structures. The groundstate structure found at $50 \% \mathrm{Ru}$ is presented in Fig. 4. One can note that in the (001) direction, we find a Ru-Mo-Rusequence which cannot occur in the more simple structures due to the periodicity requirement in this direction. An inspection of the SGPM interactions in Fig. 2 immediately shows that near the concentration of $50 \% \mathrm{Ru}$, the fourth nearest-neighbor interaction becomes important and this leads to the stabilization of the observed ground-state structure.

Further, at concentration of $63.5 \% \mathrm{Ru}$, we find another long period superstructure, in this case based on the 24 atom supercell, which we present in Fig. 5. Here, one can see that the weaker strength of the fourth nearest-neighbor interaction leads to-Mo-Ru-Ru-sequences in the (001) direction, and every alternate plane has all in-plane bonds satisfied. One can note that in this structure, all layers have either $50 \%$ or $75 \%$ composition, and thus one might expect a whole sequence of

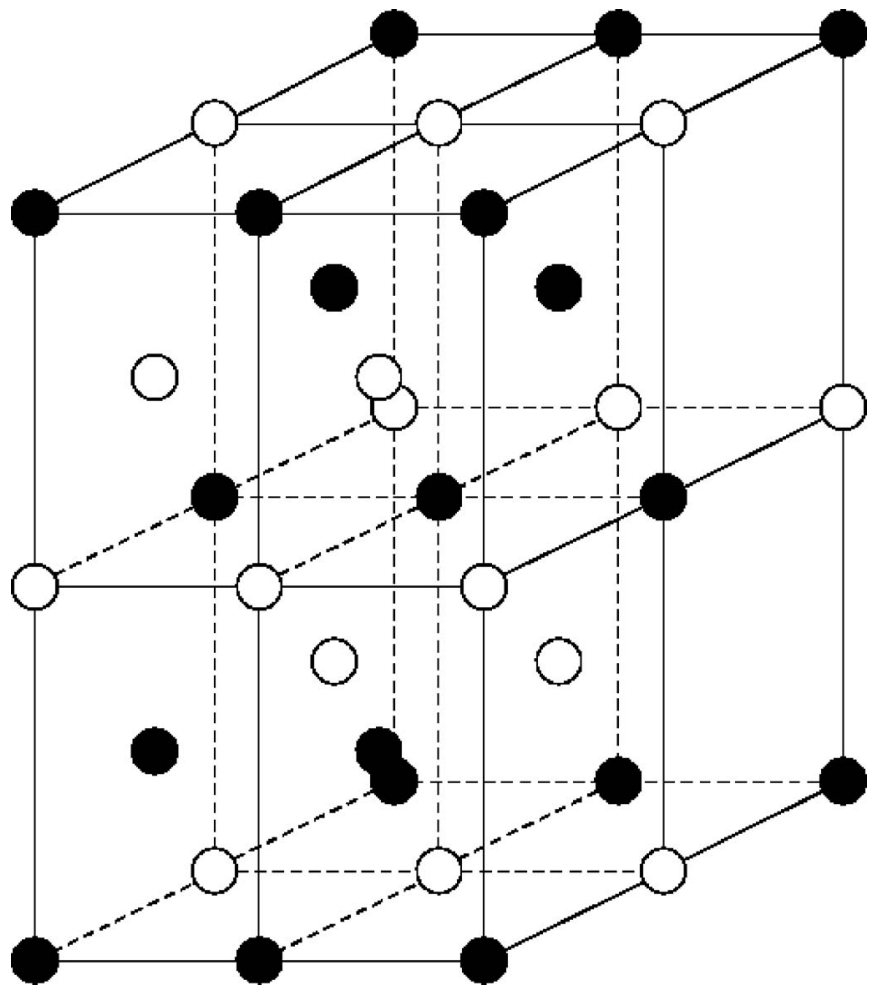

FIG. 4. 16 atom long period structure found as the lowestenergy state for $\mathrm{Mo}_{0.50} \mathrm{Ru}_{0.50}$ hcp alloy. White atoms are $\mathrm{Ru}$ and black atoms denote Mo.

the structures composed of these planes. This is similar to the adaptive structures found for the fcc lattice in Ref. 46.

\section{MIXING ENTHALPIES OF ORDERED STRUCTURES}

\section{A. Ordered structures on the hcp underlying crystal lattice}

Figure 6 shows the calculated mixing enthalpy for random Mo-Ru hcp alloy, as well as for ordered $D O_{19}$ compound at $75 \% \mathrm{Ru}$, and partially ordered $D O_{19}$ alloys at other concentrations. We also show the mixing enthalpies for partially ordered $B 19$ alloys and for the structures obtained as a result of the ground-state search described in the previous section. All the results are obtained by the direct electronic structure calculations using the EMTO method. Note that our calculated mixing enthalpy for the $D O_{19}$ structure agrees well with that presented in Ref. 5, where the latter is the lowest-energy structure for the corresponding concentration interval. As can be seen in Fig. 6, we find several long period superstructures from the ground-state search which are lower in energy than the ordered or partially ordered $D O_{19}$ alloys. Especially, this is true for concentrations between $50 \%$ and $75 \% \mathrm{Ru}$.

We can also see that even with this complete ordering, the calculated results are very far from the experimental curve, at least by $4 \mathrm{mRy}$ at every point. We therefore conclude that the ordering effects cannot explain the disagreement between theoretical and experimental values of the mixing enthalpy.

\section{B. $\sigma$ phase}

In order to complete the investigation of ordered and random alloys in Mo-Ru system, we calculated mixing energy 


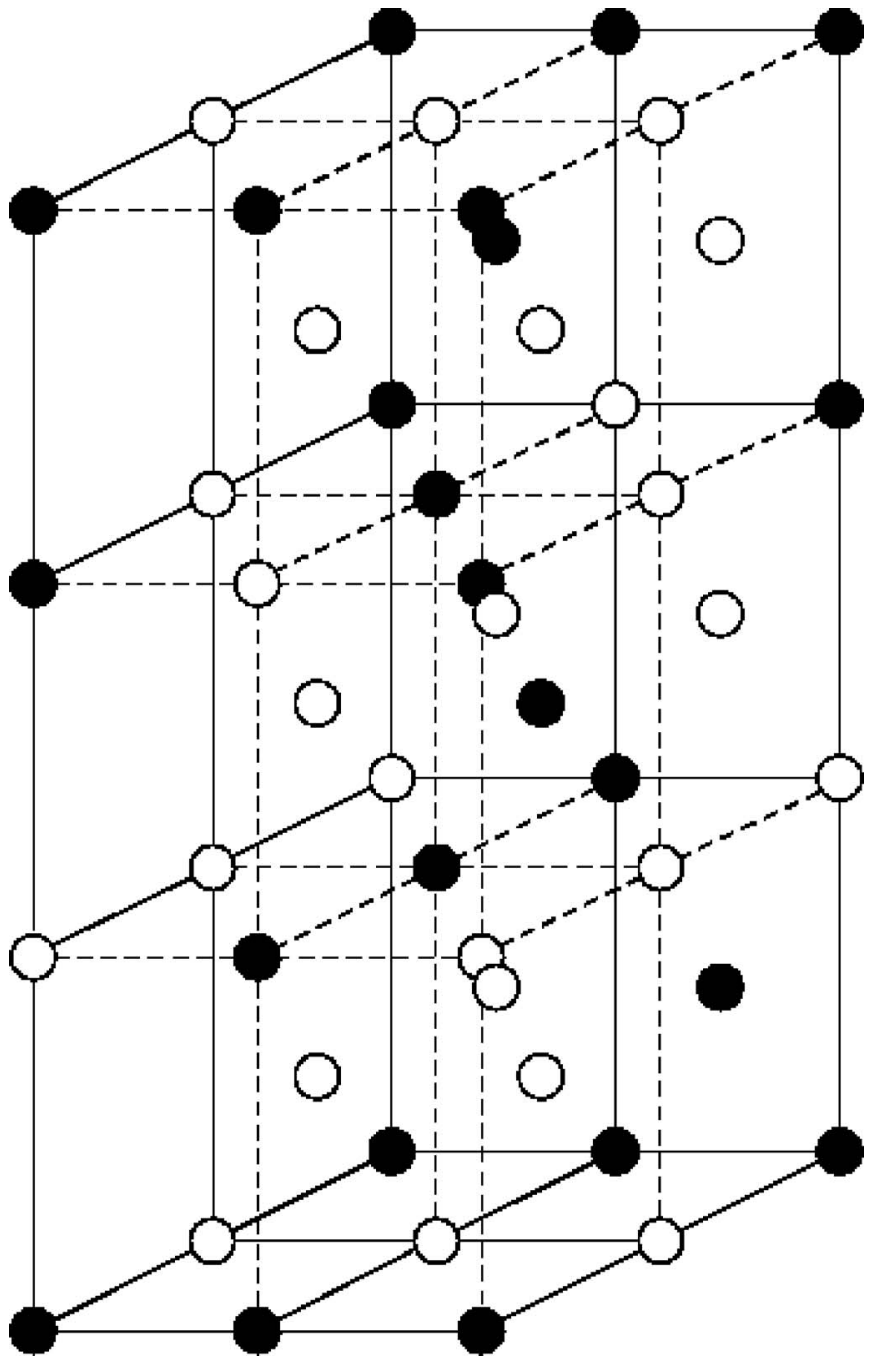

FIG. 5. 24 atom long period structure found as the lowestenergy state for $\mathrm{Mo}_{0.375} \mathrm{Ru}_{0.625}$. White atoms are $\mathrm{Ru}$ and black atoms denote Mo.

for the $\sigma$ phase. This is the only compound which appears at Mo-Ru phase diagram. The $\sigma$ phase has a very complicated structure consisting of five sublattices, two bcc-like and three fcc-like. Using the PAW method as implemented in VASP, we have carried out a complete search of the possible ground states for the $\sigma$ phase. The results will be presented elsewhere. Here, we concentrate on the $\sigma$ phase with the most stable sublattice occupation. In Fig. 6 the one lowest in enthalpy can be seen, corresponding to a Mo concentration of $67 \%$, or two-thirds. It is understandable that this should be the one with the lowest enthalpy, since the fcc-like sublattices are all filled with $\mathrm{Ru}$, which is quite stable in the fcc structure, and all bcc-like sublattices are filled with Mo, which has bec as ground-state structure. The enthalpy for this $\sigma$-phase member is $1.181 \mathrm{mRy}$. This is very reasonable, since the phase diagram ${ }^{41}$ shows that the $\sigma$ phase becomes stable first around temperature $1400 \mathrm{~K}$. At $0 \mathrm{~K}$, the $\sigma$ phase is unstable, in agreement with a positive value of the mixing enthalpy obtained in this study. However, the value of the mixing enthalpy is relatively small, and this suggests that the $\sigma$ phase can be stabilized by temperature. ${ }^{48}$ Note also that the

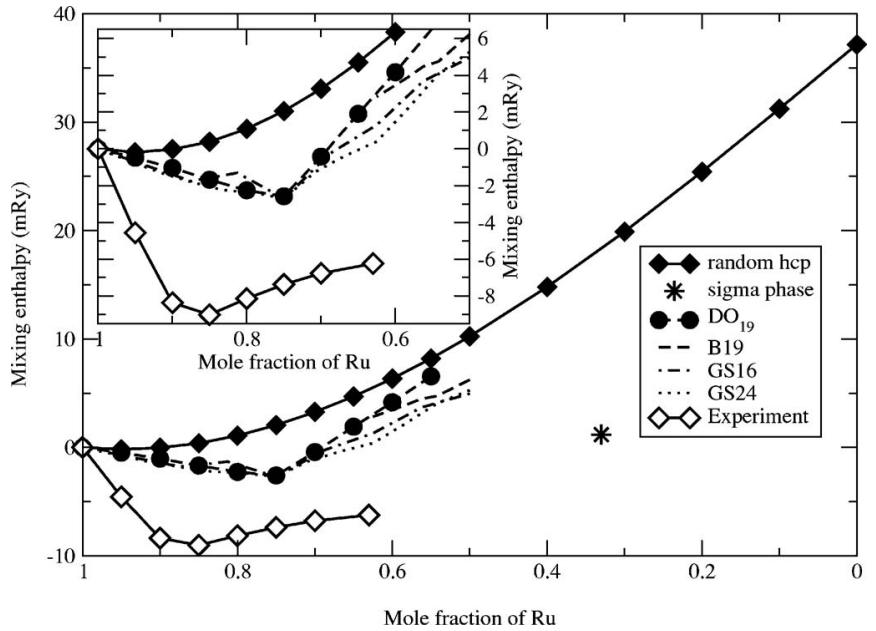

FIG. 6. Calculated mixing enthalpies of completely random hcp $\mathrm{Ru}_{x} \mathrm{Mo}_{1-x}$ alloy (filled diamonds, solid line), partially ordered $D O_{19}$ (filled circles), and $B 19$ (dashed line) alloys, as well as for the lowest-energy structures based on 16 (dot-dashed line) and 24 (dotted line) atom supercells at each concentration obtained as a result of the ground-state search described in Sec. IV. Calculated mixing enthalpy for the most stable $\sigma$ phase is shown by the star. Experimental results from Ref. 41 (open diamonds) are also presented. The inset magnifies the Ru-rich part of the graph.

stoichiometry of the $\sigma$ phase obtained in our calculations agrees with experiment.

\section{DISCUSSION}

It is generally believed that first-principles electronic structure calculations within DFT allow one to obtain mixing enthalpies for alloys and compounds in good agreement with experiment (with accuracy of the order of 1-2 mRy/atom). Therefore, ab initio simulations are considered as a reliable tool for predicting total energies of metallic systems. ${ }^{5} \mathrm{~A}$ case study of Mo-Ru system carried out in this work shows that there seem to be no way to reconcile the theoretically calculated mixing enthalpies with the available experimental results. Although it is possible to get quite close to the experimental curve by using the Mo hcp-bcc structural energy difference as a free parameter ${ }^{7,8}$ and then use the CALPHAD value for the latter, there is no obvious reason for using this method from the point of view of the electronic structure theory. One must also note that even in that case, no reasonable (non-negative) value of $E_{\text {struc }}^{\mathrm{Mo}}$ can bring the calculated mixing enthalpy into agreement with the experimental curve in the very dilute limit.

While appreciating the limitations of the first-principles DFT methodology, we would like to point out that the disagreement does not have to be exclusively due to the failure of the theory. In particular, the experimental curvature around a concentration of $85 \% \mathrm{Ru}$ is quite different from our theoretical results, and it also seem to be somewhat odd if one compares this concentration behavior with mixing enthalpies in other random alloys. ${ }^{49}$ The very reasonable values of the formation energy for the $\sigma$ phase may be an indication that theoretical results can be trusted, and this in its turn 
might suggest that the experimental results are questionable.

As a matter of fact, the experiments are very hard to perform due to the fact that Mo is a refractive metal. Therefore, it reacts with other elements only at very high temperatures and even then the diffusion time is very long for the alloy. Another big problem is an oxidation of Mo, ${ }^{50}$ which makes the use of small pellets very troublesome. Nevertheless, a new experiment would be highly interesting and might also be instrumental in resolving the debate regarding the Mo structural energy difference.

\section{CONCLUSIONS}

We have performed $a b$ initio calculations of the mixing enthalpies of completely random hcp, fcc, and bcc Mo-Ru alloys. We have further calculated the mixing enthalpies of partially ordered and ordered structures for the hcp lattice and for the $\sigma$ phase. We have estimated the relaxation energies and find that they are not important on the energy scale of the problem. We have further performed a ground-state search for a binary alloy based on the hcp lattice and found, similar to the results of recent works on the fcc and bcc lattices, a series of adaptive ground-state structures below the tie line of the simple compounds. In this case, the origin lies in a large chemical interaction between next-nearest- neighbor (111) planes. Using these ordered structures as well as the partially ordered alloys, we can make an estimation of the ordering effects in the alloy, and find those quite small as well, on the energy scale relevant for the problem. The collected results indicate that neither local lattice relaxations nor chemical ordering would allow us to reconcile the theoretically calculated mixing enthalpies with the experimental values. A new experimental effort would be highly interesting, both in that it will be used for a verification of the theoretical calculations, and also in that it might help in shedding new light on the problem with the discrepancies between $a b$ initio and CALPHAD values for the structural energy difference of Mo.

\section{ACKNOWLEDGMENTS}

We would like to thank S. I. Simak for the help in constructing the special quasirandom structure used in this work. Useful discussions with P. A. Korzhavyi and T. Burkert regarding the $\sigma$-phase calculations are gratefully acknowledged. Valuable discussions with and help from J. C. Gachon are also acknowledged. This work was supported by the Swedish Research Council (VR) and the Swedish Foundation for Strategic Research (SSF). Most of the simulations were carried out at the Swedish National Infrastructure for Computing (SNIC).
*Electronic address: andreask@ifm.liu.se

${ }^{1}$ P. Hohenberg and W. Kohn, Phys. Rev. 136, B864 (1964).

${ }^{2}$ W. Kohn and L. J. Sham, Phys. Rev. 140, A1133 (1965).

${ }^{3}$ V. Blum and A. Zunger, Phys. Rev. B 72, 020104(R) (2005).

${ }^{4}$ P. E. A. Turchi, V. Drchal, and J. Kudrnovsky, Phys. Rev. B 74, 064202 (2006).

${ }^{5}$ S. Curtarolo, D. Morgan, and G. Ceder, CALPHAD: Comput. Coupling Phase Diagrams Thermochem. 29, 163 (2005).

${ }^{6}$ L. Kaufman and H. Bernstein, Computer Calculations of Phase Diagrams (Academic, New York, 1970).

${ }^{7}$ A. E. Kissavos, S. Shallcross, V. Meded, L. Kaufman, and I. A. Abrikosov, CALPHAD: Comput. Coupling Phase Diagrams Thermochem. 29, 17 (2005).

${ }^{8}$ D. Shin, R. Arroyave, Z.-K. Liu, and A. Van de Walle, Phys. Rev. B 74, 024204 (2006).

${ }^{9}$ P. E. A. Turchi, I. A. Abrikosov, B. Burton, S. G. Fries, G. Grimvall, L. Kaufman, P. A. Korzhavyi, V. Rao Manga, M. Ohno, A. Pisch, A. Scott, and W. Zhang, CALPHAD: Comput. Coupling Phase Diagrams Thermochem. 31, 4 (2007).

${ }^{10}$ For a review see Challenges in Plutonium Science, Los Alamos Science No. 26, edited by N. G. Cooper (Los Alamos National Laboratory, Los Alamos, 2000).

${ }^{11}$ Y. Wang, S. Curtarolo, C. Jiang, R. Arroyave, T. Wang, G. Ceder, L.-Q. Chen, and Z.-K Liu, CALPHAD: Comput. Coupling Phase Diagrams Thermochem. 28, 79 (2004).

${ }^{12}$ G. Grimvall, M. Thiessen, and A. F. Guillermet, Phys. Rev. B 36, 7816 (1987).

${ }^{13}$ L. Kaufman, CALPHAD: Comput. Coupling Phase Diagrams Thermochem. 25, 141 (2001).
${ }^{14}$ J. O. Anderson, A. F. Guillermet, and P. Gustafssen, CALPHAD: Comput. Coupling Phase Diagrams Thermochem. 11, 361 (1987).

${ }^{15}$ H. L. Skriver, Phys. Rev. B 31, 1909 (1985).

${ }^{16}$ J. A. Moriarty, Phys. Rev. B 45, 2004 (1992).

${ }^{17}$ B. K. Godwal and R. Jeanloz, Phys. Rev. B 41, 7440 (1990).

${ }^{18}$ P. Soderlind, R. Ahuja, O. Eriksson, B. Johansson, and J. M. Wills, Phys. Rev. B 49, 9365 (1993).

${ }^{19}$ E. A. Smirnova, R. Ahuja, Yu. Kh. Vekilov, B. Johansson, Y. K. Vohra, and I. A. Abrikosov, Phys. Rev. B 66, 024110 (2002).

${ }^{20}$ K. Persson, M. Ekman, and G. Grimvall, Phys. Rev. B 60, 9999 (1999).

${ }^{21}$ I. A. Abrikosov, A. V. Ruban, B. Johansson, and H. L. Skriver, Comput. Mater. Sci. 10, 302 (1998).

${ }^{22}$ P. Olsson, I. A. Abrikosov, L. Vitos, and J. Wallenius, J. Nucl. Mater. 321, 84 (2003).

${ }^{23}$ Lectures on Methods of Electronic Structure Calculations, edited by O. K. Andersen, O. Jepsen, and G. Krier, in V. Kumar, O. K. Andersen, and A. Mookerjee (World Scientific, Singapore, 1994), pp. 63-124.

${ }^{24}$ O. K. Andersen, C Arcangeli, R. W. Tank, T. Saha-Dasgupta, G. Krier, O. Jepsen, and I. Dasgupta, in Tight-Binding Approach to Computational Materials Science, MRS Symposia Proceedings Vol. 491, edited by P. E. A. Turchi, A. Gonis, and L. Colombo (Materials Research Society, Pittsburgh 1998), p. 3.

${ }^{25}$ L. Vitos, Phys. Rev. B 64, 014107 (1996).

${ }^{26}$ L. Vitos, J. Kollár, and H. L. Skriver, Phys. Rev. B 55, 13521 (1997).

${ }^{27}$ J. Kollár, L. Vitos, and H. L. Skriver, Electronic Structure and 
Physical Properties of Solids: The Uses of the LMTO Method, Lecture Notes in Physics, edited by H. Dreyss (Springer-Verlag, Berlin, 2000), pp. 85-113.

${ }^{28}$ J. S. Faulkner, Prog. Mater. Sci. 27, 1 (1982).

${ }^{29}$ I. A. Abrikosov, Y. H. Vekilov, P. A. Korzhavyi, A. V. Ruban, and L. E. Shilkrot, Solid State Commun. 83, 867 (1992).

${ }^{30}$ P. A. Korzhavyi, A. V. Ruban, I. A. Abrikosov, and H. L. Skriver, Phys. Rev. B 51, 5773 (1995).

${ }^{31}$ A. V. Ruban, S. Shallcross, S. I. Simak, and H. L. Skriver, Phys. Rev. B 70, 125115 (2004).

${ }^{32}$ L. Vitos, I. A. Abrikosov, and B. Johansson, Phys. Rev. Lett. 87, 156401 (2001).

${ }^{33}$ A. E. Kissavos, S. I. Simak, P. Olsson, L. Vitos, and I. A. Abrikosov, Comput. Mater. Sci. 35, 1 (2006).

${ }^{34}$ J. P. Perdew, K. Burke, and M. Ernzerhof, Phys. Rev. Lett. 77, 3865 (1996).

${ }^{35}$ P. E. Blöchl, Phys. Rev. B 50, 17953 (1994).

${ }^{36}$ G. Kresse and J. Hafner, Phys. Rev. B 48, 13115 (1993).

${ }^{37}$ G. Kresse and J. Furthmüller, Comput. Mater. Sci. 6, 15 (1996).

${ }^{38}$ G. Kresse and J. Furthmüller, Phys. Rev. B 54, 11169 (1996).

${ }^{39}$ A. Zunger, S. H. Wei, L. G. Ferreira, and J. E. Bernard, Phys. Rev. Lett. 65, 353 (1990).

${ }^{40}$ I. A. Abrikosov, S. I. Simak, B. Johansson, A. V. Ruban, and H.
L. Skriver, Phys. Rev. B 56, 9319 (1997).

${ }^{41}$ H. Kleykamp, J. Nucl. Mater. 167, 49 (1989).

${ }^{42}$ A. V. Ruban, S. I. Simak, S. Shallcross, and H. L. Skriver, Phys. Rev. B 67, 214302 (2003).

${ }^{43}$ F. Ducastelle, Order and Phase Stability in Alloys (NorthHolland, Amsterdam, 1991).

${ }^{44}$ A. V. Ruban and H. L. Skriver, Phys. Rev. B 66, 024201 (2002).

${ }^{45}$ A. V. Ruban, S. I. Simak, P. A. Korzhavyi, and H. L. Skriver, Phys. Rev. B 66, 024202 (2002).

${ }^{46}$ M. Sanati, L. G. Wang, and A. Zunger, Phys. Rev. Lett. 90, 045502 (2003).

${ }^{47}$ R. Drautz, A. Diaz-Ortiz, M. Fähnle, and H. Dosch, Phys. Rev. Lett. 93, 067202 (2004).

${ }^{48}$ P. A. Korzhavyi, B. Sundman, M. Selleby, and B. Johansson, Atomic, Electronic, and Magnetic Structure of Iron-Based Sigma-Phases, Materials Research Society Symposia Proceedings Vol. 842 (Materials Research Society, Pittsburgh, 2005), p. S4.10.1.

${ }^{49}$ R. Hultgren, P. D. Desai, D. T. Hawkins, M. Gleiser, and K. K. Kelley, Selected Values of Thermodynamic Properties of Binary Alloys (American Society for Metals, Metals Park, OH, 1973).

${ }^{50}$ J. C. Gachon (private communications). 